\title{
Exploring in-situ viral infection with multi-modal cryogenic correlative FLM- FIB/SEM/Cryo-ET for vitrified mammalian cells
}

\author{
Jae Yang ${ }^{1}$, Matthew Larson ${ }^{2}$, Joseph $\mathrm{Kim}^{3}$ and Elizabeth Wright ${ }^{1}$
}

${ }^{1}$ Department of Biochemistry, University of Wisconsin-Madison, Madison, Wisconsin, United States, ${ }^{2}$ CryoElectron Microscopy Research Center, Department of Biochemistry, University of Wisconsin, Madison, WI, USA, Madison, Wisconsin, United States, ${ }^{3}$ Department of Chemistry, University of Wisconsin-Madison, Madison, Wisconsin, United States

Cryo-electron tomography (cryo-ET) has emerged as a powerful tool to provide in-situ detailed, nanometer to sub-nanometer level three-dimensional (3D) information on cellular ultrastructure and macromolecular complexes during host-virus interactions [1]. However, a few challenges remain when using cryo-ET for in situ structural studies, that include difficulties in locating and identifying features of interest within complex cellular surroundings and the thickness of some samples. Electrons are prone to inelastic scattering in biological samples thicker than $300 \mathrm{~nm}$, e.g., regions away from thin cell periphery of eukaryotic cells, this results in image blurring and decreased resolution. To visualize them, cryo-focused ion beam (FIB) milling coupled with scanning electron microscopy (cryo-FIB/SEM) has been used to generate thin lamella from vitrified eukaryotic cells, making a wide variety of exciting biological events accessible for cryo-ET [2]. Cryocorrelative light and electron microscopy (cryo-CLEM), the coupling of fluorescent light microscopy (FLM) and transmission electron microscopy (TEM), adds temporal and spatial information from FLM to cryo-ET, facilitating identification of rare or dynamic events during viral infection [3]. Thus, multi-modal and -scale microscopy under cryo-conditions has been explored to locate fluorescent regions of interest by cryo-FLM, in a thin lamella of the vitrified eukaryotic cell produced by cryo-FIB/SEM, for high-resolution cryo-ET study [4].

Respiratory syncytial virus (RSV), an enveloped RNA virus of the Pneumoviridae family, is a leading cause of serious lower respiratory tract illness in infants and immuno-suppressed adults [5]. Recent studies show that mitochondrial organization and its functions have been usurped by RSV to facilitate virus production in infected cells [6]. While high-resolution live-cell FLM quantitative imaging [6] and conventional TEM on resin-embedded cells [7] have greatly advanced our understanding of mitochondria morphology, details in the ultrastructural features of mitochondria and the in-situ morphological changes mitochondria undergo during RSV infection remain unclear. Here, we present a correlated multi-modal pipeline to explore native mitochondrial ultrastructure in RSV-infected cells by coupling cryo-FLM and FIB/SEM of vitrified thin lamella, then visualized by high resolution cryo-ET, using CorRelator [8], an interactive and cross-platform software we developed for flexible real-time high-precision cryo-correlative microscopy.

Briefly, HeLa cells were grown on Quantifoil gold grids and infected with RSV rA2-mK+. Samples were also stained with live-cell MitoTrackerGreen. The grids were imaged with a Leica DMi8 widefield fluorescent microscope at room-temperature prior to plunge freezing. Single-sided, back blotting, and plunge-freezing was performed using a Leica EMGP. The vitrified samples were imaged by a Leica EM Cryo-CLEM microscope system. Correlated cryo-FIB/SEM was then conducted to generate uniform thin lamellas (thickness of 200 $\mathrm{nm})$ of the mitochondria-rich region inside the infected or uninfected/mock cells. The lamellas were subsequently imaged by a Leica EM Cryo-CLEM system to verify the milling location. We then collected tilt series of these vitrified lamellas on a Titan Krios or Talos Arctica equipped with a Gatan BioQuantum filter and K3 direct electron detector, using SerialEM [9]. Throughout the whole workflow, CorRelator was flexibly applied for correlation between cryo-FLM and cryo-FIB/SEM and cryo-ET for real-time high-precision cryocorrelative ET data collection. Tomographic reconstructions were prepared with IMOD [10] and segmentation using EMAN2.2 neural network [11]. 
Cryo-FLM and cryo-FIB/SEM correlation allows us to locate mitochondria-rich regions in RSV-infected and uninfected cells and generate thin lamella suitable for high resolution cryo-ET studies (Figure 1, top panel AG). Post-FIB/SEM cryo-CLEM further confirmed the milled region of interest (Figure 1, bottom panel A-C). Correlated cryo-ET of targeted lamella (Figure 1, bottom panel D-E) show the impact of macromolecular and vesicular crowding in the cytoplasm along with the presence of the mitochondrial targets near microtubules. ATP-synthase complexes embedded in the cristae membranes (red arrowheads, Figure 2) were highlighted. This workflow presented here demonstrates the flexibility of a multi-modal cross-platform microscopy. Future developments of CorRelator include high-precision 3D correlation between cryo-FLM and cryo-FIB/SEM for subsequent cryo-ET data collection.

This research was supported by funds from the University of Wisconsin-Madison, Morgridge Institute for Research, and National Institutes of Health (R01GM104540, R01GM104540-03S1, R01 GM114561, and U24 GM139168) to E.R.W. All EM data was collected at the Cryo-EM Research Center, Department of Biochemistry, University of Wisconsin-Madison.

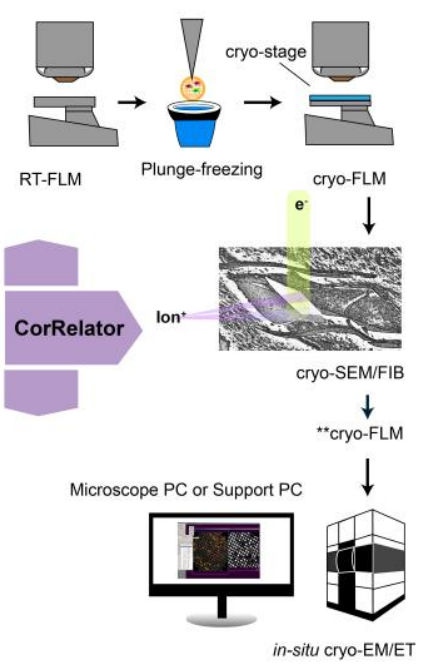

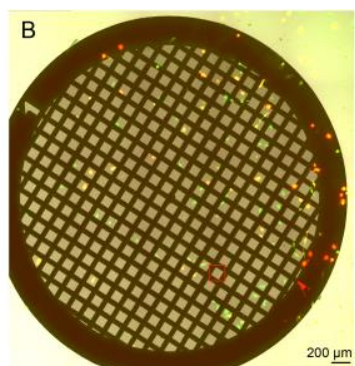

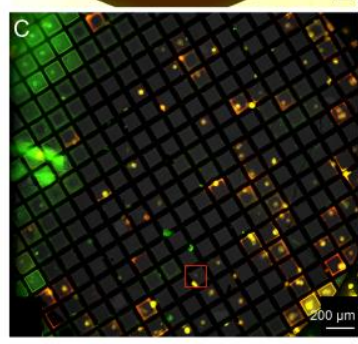

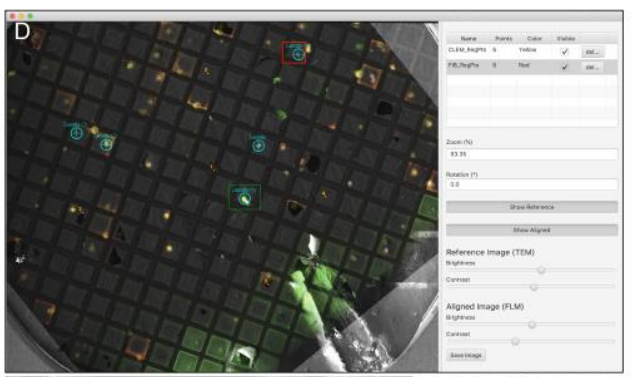

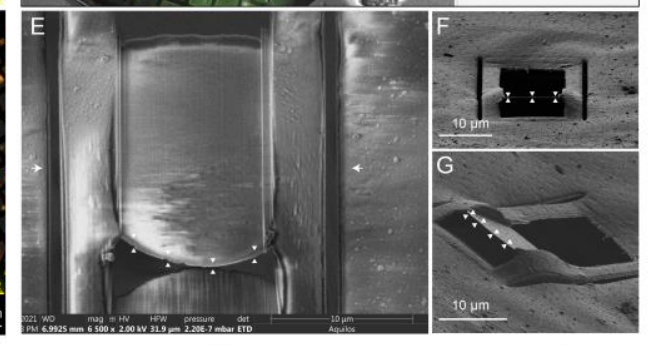

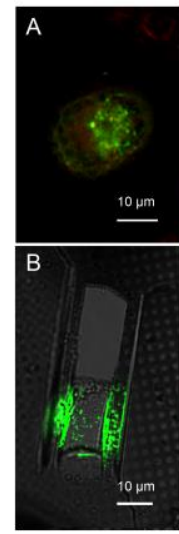
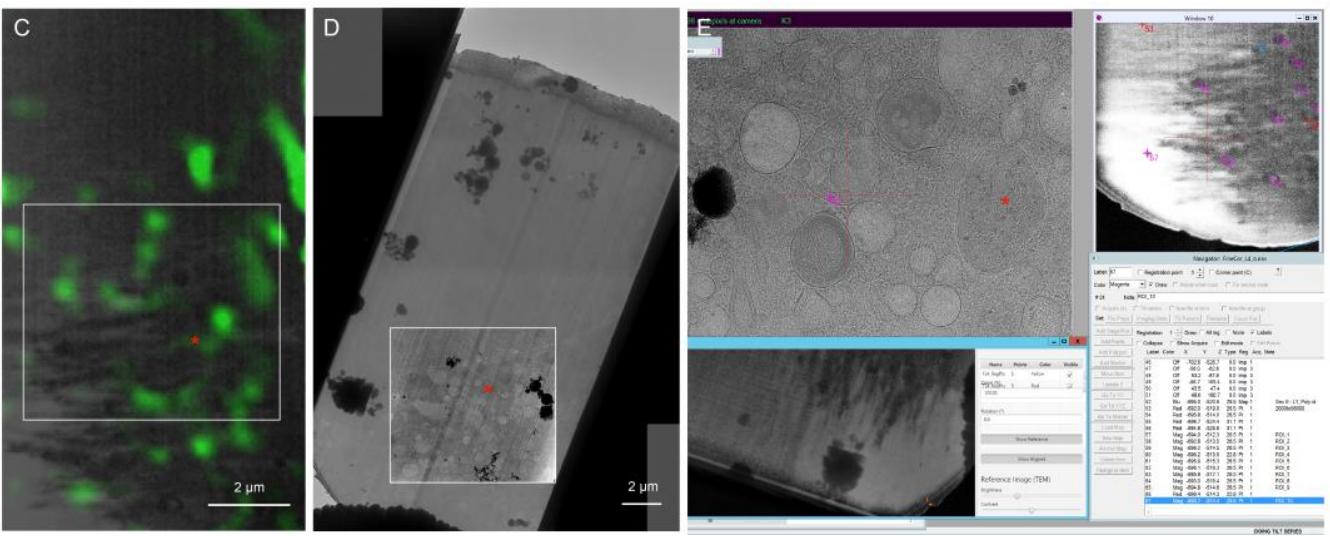

Figure 1. Figure 1. Multi-modal cryo-CLEM-FIB/SEM -ET lamellae of HeLa cells infected by RSV (red), stained to study mitochondria (green). Top Panel: (A) A diagram of CorRelator-facilitated multi-modal correlative microscopy. (B) Merged live-cell fluorescent microscopy (FLM) grid montage of RSV rA2-mK+ infected HeLa cells. (C) Cryo-FLM grid montage of grid in (B) after plunge-freezing. (D) GUI Screenshot of correlated cryo-FLM (C) and cryo-SEM maps using CorRelator to guide the milling process. Red boxes in (BD) are region of interest. (E-G) Cryo-FIB/SEM thin lamellae of the target cells (red boxed cells in B-D). Milled 
cells ( 200 nm thickness) visualized by cryo-SEM (2eV, E) and cryo-FIB (F and its 45-degree rotation $\mathrm{G})$. The deposited Pt layer (white arrow heads) is distinguishable from the vitrified cell on the front edge of the lamellae. Micro-expansion joints (white arrows) are labeled. Bottom Panel: (A-C) Cryo-FLM of the lamellae in (Top: E-G) to show the cell before milling (A) and post-milling (B), and the superimposition (C) of cryoFLM (B) and lamellae (Top: E). (D-E) Correlative real-time cryo-ET of the lamellae. White boxed area (D where high pass-filtering applied) and red asterisk show the mitochondria region of interest. (E) Screenshot of real-time correlative cryo-ET collection of the same lamellae using CorRelator and SerialEM.
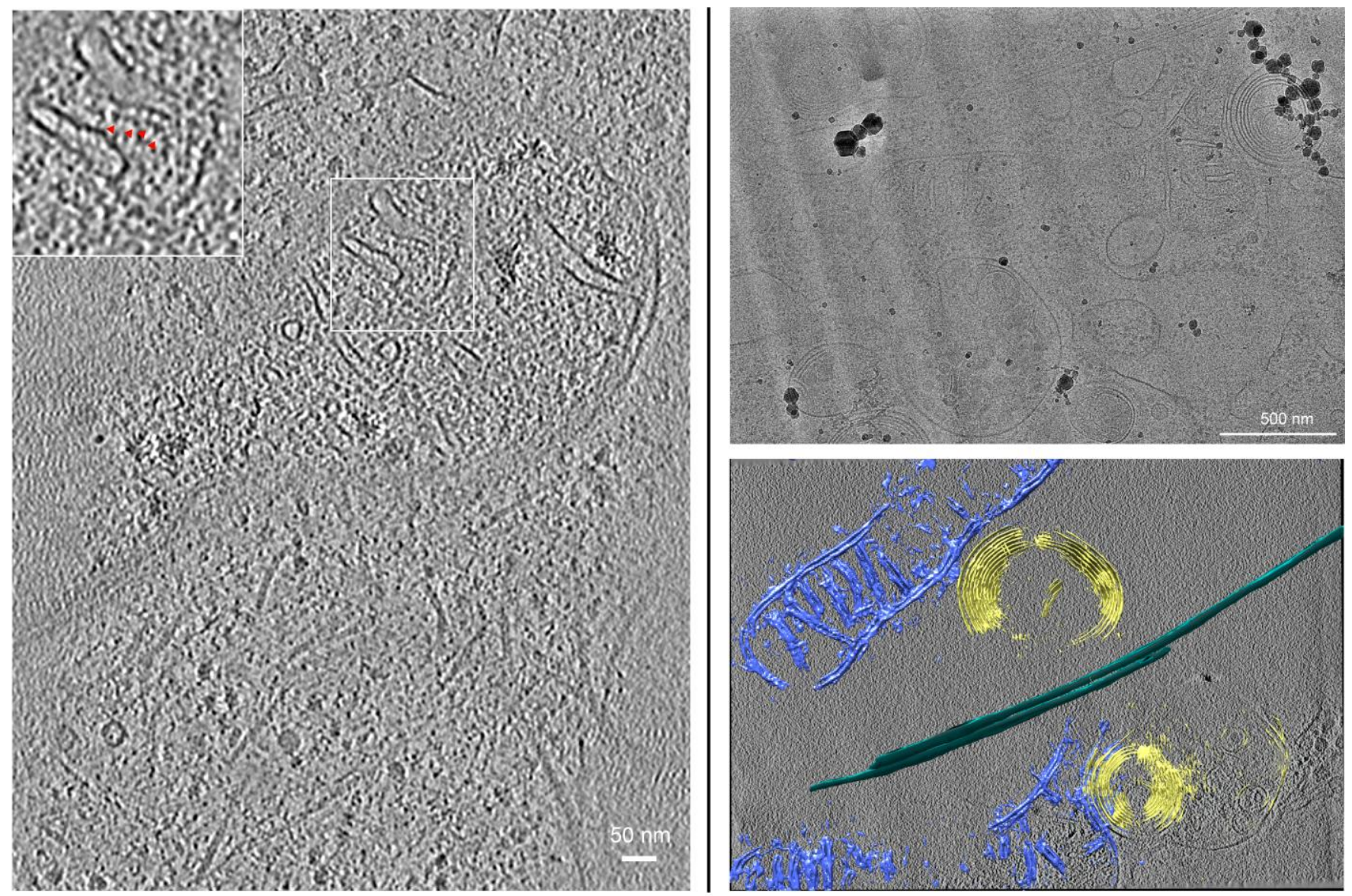

Figure 2. Figure 2. Cryo-EM imaging and cryo-ET on lamellas. (Left) Tomographic slice through a mitochondria-rich region in RSV-infected cells, reconstructed with IMOD, which shows the mitochondria highlighting ATP-synthase complexes embedded in the cristae membranes (red arrowheads). (Right) cryo-EM image display mitochondria and the crowded surrounding cellular context (top) and the segmentation (bottom) highlighting mitochondria (blue), multilamellar vesicles (yellow) and microtubules (green) 
1. Ke, Z., et al., Structures and distributions of SARS-COV-2 spike proteins on intact virions. Nature, 2020. 588(7838): p. 498-502.

2. Rigort, A., et al., Chapter 14 - Integrative Approaches for Cellular Cryo-electron Tomography: Correlative Imaging and Focused Ion Beam Micromachining, in Methods in Cell Biology, T. Müller-Reichert and P. Verkade, Editors. 2012, Academic Press. p. 259-281.

3. Hampton, C.M., et al., Correlated fluorescence microscopy and cryo-electron tomography of virusinfected or transfected mammalian cells. Nature Protocols, 2017. 12(1): p. 150-167.

4. Wu, G.H., et al., Multi-scale 3D Cryo-Correlative Microscopy for Vitrified Cells. Structure, 2020. 28(11): p. 1231-1237.e3.

5. Nair, H., et al., Global burden of acute lower respiratory infections due to respiratory syncytial virus in young children: a systematic review and meta-analysis. The Lancet, 2010. 375(9725): p. 1545-1555.

6. Hu, M., et al., Respiratory syncytial virus co-opts host mitochondrial function to favour infectious virus production. elife, 2019. 8: p. e42448.

7. Karbowski, M. and R.J. Youle, Dynamics of mitochondrial morphology in healthy cells and during apoptosis. Cell Death \& Differentiation, 2003. 10(8): p. 870-880.

8. Yang, J.E., et al., CorRelator: Interactive software for real-time high precision cryo-correlative light and electron microscopy. J Struct Biol, 2021: p. 107709.

9. Mastronarde, D.N., Automated electron microscope tomography using robust prediction of specimen movements. Journal of Structural Biology, 2005. 152(1): p. 36-51.

10. Kremer, J.R., D.N. Mastronarde, and J.R. McIntosh, Computer visualization of three-dimensional image data using IMOD. J Struct Biol, 1996. 116(1): p. 71-6.

11. Chen, M., et al., Convolutional neural networks for automated annotation of cellular cryo-electron tomograms. Nature methods, 2017. 14(10): p. 983-985. 